\title{
Synergistic phase behaviors of phytosterols and phytosterol esters in medium chain triglycerides
}

\author{
Shuai Zhou ${ }^{1}$, Yue Sun ${ }^{1}$, Yong Wang ${ }^{1}$, and Yuanrong Jiang ${ }^{1}$ \\ ${ }^{1}$ Wilmar Shanghai Biotechnology Research and Development Center Co Ltd
}

October 25, 2021

\begin{abstract}
Free phytosterols (PS) and phytosterol esters (PSE) usually coexist in natural oils, which significantly affect the crystallization behavior of oil. However, the phase behaviors of PS/PSE mixtures in oils and their roles on the crystallization behavior of oil are still unclear. In this study, the dissolution and crystallization behaviors of PS/PSE mixtures in medium chain triglyceride (MCT) were studied, and the synergistic mechanism of PS/PSE mixtures on the crystallization behaviors of MCT was further clarified. The results showed that when the mass ratio of PS: PSE was greater than 3: 7, PS/PSE mixtures recrystallized a new urchin-like crystal structure, which was different from PS spherical or PSE plate-like crystal structure, in MCT. The new urchin-like PS/PSE crystal had a larger surface area, and its melting point was between PS and PSE. It also showed the synergistic ability to promote the crystallization nucleation of MCT. When the total content of PS and PSE was 1 wt\%, and the mass ratio of PS: PSE was 5: 5, the crystallization peak temperature of PS/PSE/MCT was $12{ }^{\circ} \mathrm{C}$ higher than that of virgin MCT, $2.5^{\circ} \mathrm{C}$ higher than that of PSE/MCT, and $10.3{ }^{\circ} \mathrm{C}$ higher than that of PS/MCT. These results not only provide a method for obtaining new crystal structure of phytosterols, but also have great significance for controlling the crystallization behaviors of oils by precisely adjusting the nutritional composition of oils.
\end{abstract}

\section{Synergistic phase behaviors of phytosterols and phytosterol esters in medium chain triglyce-} rides

Shuai Zhou*, Yue Sun*, Yong Wang, Yuanrong Jiang

Division of Culinary Oils, Wilmar (Shanghai) Biotechnology Research \& Development Center Co., Ltd, Shanghai, 200137, China

* Corresponding authors: Shuai Zhou; Yue Sun

E-mail addresses: zhoushuai860311@163.com (Shuai Zhou);

sunyue5@cn.wilmar-intl.com (Yue Sun);

wangyongksf@@cn.wilmar-intl.com (Yong Wang);

jiangyuanrong@cn.wilmar-intl.com (Yuanrong Jiang);

Abstract: Free phytosterols (PS) and phytosterol esters (PSE) usually coexist in natural oils, which significantly affect the crystallization behavior of oil. However, the phase behaviors of PS/PSE mixtures in oils and their roles on the crystallization behavior of oil are still unclear. In this study, the dissolution and crystallization behaviors of PS/PSE mixtures in medium chain triglyceride (MCT) were studied, and the synergistic mechanism of PS/PSE mixtures on the crystallization behaviors of MCT was further clarified. The results showed that when the mass ratio of PS: PSE was greater than 3: 7, PS/PSE mixtures recrystallized a new urchin-like crystal structure, which was different from PS spherical or PSE plate-like crystal structure, in MCT. The new urchin-like PS/PSE crystal had a larger surface area, and its melting point was between 
PS and PSE. It also showed the synergistic ability to promote the crystallization nucleation of MCT. When the total content of PS and PSE was $1 \mathrm{wt} \%$, and the mass ratio of PS: PSE was 5: 5, the crystallization peak temperature of PS/PSE/MCT was $12{ }^{\circ} \mathrm{C}$ higher than that of virgin MCT, $2.5{ }^{\circ} \mathrm{C}$ higher than that of $\mathrm{PSE} / \mathrm{MCT}$, and $10.3{ }^{\circ} \mathrm{C}$ higher than that of PS/MCT. These results not only provide a method for obtaining new crystal structure of phytosterols, but also have great significance for controlling the crystallization behaviors of oils by precisely adjusting the nutritional composition of oils.

Key words: phytosterols; phytosterol esters; medium chain triglycerides; crystallization; synergistic effect; crystal structure

\section{Introduction}

Phytosterols are natural substances with important biological functions for human body, like the lowering effect of cholesterols (Bohn et al., 2007; Goldberg et al., 2006), anti-flammatory (Hannan et al., 2020), anticancer (Shahzad et al., 2017) and immunity strengthen effect (Bouic et al., 1999) etc. It shows an increasingly popular trend in the consumer market and becomes the hot research topic in foods and oils industry.

However, due to the phase characteristics of phytosterols, their practical applications in foods and oils are limited, especially in edible vegetable oil. The solubility of phytosterols in oils is very low while its melting point is quite high (about $140-150{ }^{\circ} \mathrm{C}$ ) (Wester, 2000). Phytosterols will precipitate in oils, which may cause turbidity in the process of oil storage, thus affecting the shelf life of the products and the acceptability of the products from the perspective of consumers. The crystallization behaviors of phytosterols in vegetable oil have been reported. Vaikousi et al. (Vaikousi, Lazaridou, Biliaderis, \& Zawistowski, 2007) had studied the thermal properties, solubility characteristics and crystallization kinetics of four kinds of commercial phytosterols and their mixtures in corn oil. The results showed that the crystal structure and morphology of commercial phytosterols and their corresponding precipitates in oil were different. In order to improve the performance of phytosterols in food and oils, esterification of phytosterols with fatty acids has been studied to improve the oil solubility of phytosterols (Nguyen, Huang, \& Su, 2020; Yang, Oyeyinka, \& Ma, 2016), and the phase behavior of phytosterol esters in vegetable oils has also been reported (Daels, Foubert, \& Goderis, 2017; Vu, Shin, Lim, \& Lee, 2004). Vu et al. (Vu, Shin, Lim, \& Lee, 2004) had reported the synthesis and crystallization behavior of phytosterol esters in corn oil. The results showed that the crystallization temperature of the medium chain fatty acid sterol ester increased with the increase of acyl group carbon numbers, among which sterol laurate had the highest crystallization temperature in corn oil.

Usually, the free phytosterols (PS) and phytosterol esters (PSE) coexist in natural vegetable oil (Esche, Scholz, \& Engel, 2013; Galeano Diaz, Durán Merás, Sánchez Casas, \& Alexandre Franco, 2005; Phillips, Ruggio, Toivo, Swank, \& Simpkins, 2002), but the phase behaviors of PS/PSE mixtures in oils, and whether PS and PSE have synergistic effects on the crystallization behaviors of oils are still unclear. At present, there are only a few synergistic compounds related to phytosterols, which have been widely studied, such as $\gamma$-oryzanol and $\beta$-sitosterol (Sawalha et al., 2012; Sawalha, Venema, Bot, Flöter, \& van der Linden, 2011), monoglyceride and phytosterol (Bin Sintang, Rimaux, Van de Walle, Dewettinck, \& Patel, 2017). Therefore, it is very important to elucidate the dissolution and crystallization behaviors of PS/PSE mixtures in oils for regulating the shelf life of oils and evaluating the possibility of oil-based delivery system as a functional component of foods.

In this study, the dissolution and crystallization behaviors of PS/PSE mixtures in medium chain triglycerides $(\mathrm{MCT})$ were investigated systematically, and the synergistic mechanism of PS/PSE mixtures on the crystallization behaviors of MCT was further clarified in depth. This will be of great significance to control the quality of oils through accurately adjusting the nutritional composition of oil.

\section{Materials and methods}

\subsection{Materials}

MCT was purchased from Dubois Natural Esters Sdn. Bhd. (Malaysia), which was composed of two kinds of fatty acids (54.6\% of C8:0 and 45.4\% of C10:0). PS, brand PS-95 (95 wt\% free phytosterol, in which beta 
sitosterol was $42.2 \mathrm{wt} \%$, stigmasterol was $26.7 \mathrm{wt} \%$, campesterol was $25.6 \mathrm{wt} \%$, brassicasterol was 0.9 wt $\%$, others were $4.6 \%$ ) with melting point of $130^{\circ} \mathrm{C}$, was purchased by Wilmar Spring Fruit Nutrition Products (Jiangsu) Co., Ltd. PSE, brand Vegapure $95 \mathrm{E}$ with melting point of about $70^{\circ} \mathrm{C}$, was provided by BASF (China) Co., Ltd. Vegapure $95 \mathrm{E}$ were obtained by esterification of free plant sterols derived from vegetable oils, and with fatty acids obtained from vegetable oils. The sterol composition of vegapure $95 \mathrm{E}$ was about $77.7 \mathrm{wt} \%$ for beta sitosterol, $9.9 \mathrm{wt} \%$ for sitosterol, $7.6 \mathrm{wt} \%$ for campesterol and $4.8 \mathrm{wt} \%$ for others. All materials were used as received.

\subsection{Sample preparation}

A certain amount of accurately weighed PS and PSE was added into MCT to make PS/MCT, PSE/MCT and PS/PSE/MCT mixtures. The mixtures were stirred and heated to $130{ }^{\circ} \mathrm{C}$ for 5 min to ensure that PS and PSE were fully dissolved in MCT, and then stored samples at room temperature. The naming rule of samples was a-PSxPSEy, specifically, the total addition of PS and PSE was a wt\%, and the mass ratio of PS: PSE was x: y. For example, sample 1-PS5PSE5 represents that the total addition of PS and PSE was 1 wt $\%$, and the mass ratio of PS: PSE was 5: 5.

\subsection{Melting and crystallization behaviors}

Differential scanning calorimetry (DSC, Q2000, TA Instruments Inc., USA) was applied to probe the nonisothermal melting and crystallization behaviors of the samples. Approximately $6 \mathrm{mg}$ sample was weighed and heated from $20^{\circ} \mathrm{C}$ to $160^{\circ} \mathrm{C}$ at a heating rate of $10^{\circ} \mathrm{C}^{*} \mathrm{~min}^{-1}$. After maintaining for 5 min to eliminate the thermal history and residual crystals, the sample was cooled down to - 65 oC at a cooling rate of 5 $\mathrm{oC}^{*} \mathrm{~min}^{-1}$. After holding for $5 \mathrm{~min}$ at $-65 \mathrm{oC}$, the sample was reheated to $160 \mathrm{oC}$ at a heating rate of 10 $\mathrm{oC}^{*} \min ^{-1}$.

\subsection{Crystal morphology}

The crystal morphologies of the samples were observed by polarized light microscope (PLM, E400, Nikon, Japan), equipped with a heating and cooling stage. The crystal morphologies of PS, PSE and PS/PSE mixtures precipitated in MCT were observed at room temperature. For obtaining the non-isothermal crystallization crystal morphologies, the samples were firstly heated to $130 \mathrm{oC}$ and held for 5 minutes to erase the thermal history and residual crystals, then cooled to - $30 \mathrm{oC}$ at a cooling rate of $5 \mathrm{oC}^{*} \mathrm{~min}^{-1}$.

\subsection{Crystal form}

The crystal forms of samples were characterized on an X-ray powder diffraction measurement (XRD, X'Pert PRO, PANalytical B.V., Netherlands). XRD patterns from $2 \vartheta 12^{\mathrm{O}}$ to $30^{\mathrm{O}}$ at $0.5^{\mathrm{O}} * \mathrm{~min}^{-1}$ with a step size of $0.02 \mathrm{o}$ were recorded at room temperature using $\mathrm{Cu} \mathrm{K} \alpha$ radiation $(\lambda=1.54056 \AA, 40 \mathrm{kV}, 40 \mathrm{~mA})$.

\subsection{Statistical analysis}

All experiments were done in triplicate. All results were expressed as mean values. Statistical analysis was performed using Origin 8.0 software (OriginLab Ltd.). One-way analysis of variance was carried out by Tukey adjustment ( $p[?]$ 0.05) to determine the significant difference between different samples.

\section{Results and discussion}

\subsection{Crystallization behaviors of PS/PSE mixtures in MCT}

The thermal behaviors of PS/PSE mixtures in MCT were measured by DSC. Fig. 1 shows the DSC cooling and heating profiles of PS/PSE mixtures with different PS: PSE mass ratios in MCT. The total concentration of PS and PSE in MCT was fixed at $5 \mathrm{wt} \%$. Fig. 1 demonstrates that the thermal behaviors of PS/PSE mixtures in MCT were significantly affected by PS: PSE mass ratios.

The DSC cooling curves of PS/PSE mixtures with different PS: PSE mass ratios in MCT are shown in Fig. 1a. When $5 \mathrm{wt} \%$ PSE was added into MCT alone (5-PS0PSE10), the crystallization peak temperatures ( $T$ сp) of PSE in MCT was - 15.9 oC, while when 5 wt\% PS was added into MCT alone (5-PS10PSE0), the $T$ cp of 
PS in MCT was - 3.3 oC, indicating that PSE had higher oil solubility than PS at the same temperature and amount, which was consistent with the research results of Vaikousi et al. (Vaikousi, Lazaridou, Biliaderis, \& Zawistowski, 2007) and Vu et al. (Vu, Shin, Lim, \& Lee, 2004). With the increase of PS: PSE mass ratio, the $T$ cp of PS/PSE mixtures in MCT first decreased and then increased. When PS: PSE mass ratio was 3: 7, the $T_{\text {cp }}$ of PS/PSE mixtures in MCT reached its lowest, which was $-21.7 \mathrm{oC}$. The change was chiefly caused by the fact that the oil solubility of PS was lower than that of PSE. When the content of PS in PS/PSE mixtures was less, the content of PSE also decreased, thus the $T$ cp of PS/PSE mixtures in MCT decreased; with the increase of PS content, the $T$ cp of PS/PSE mixtures in MCT increased gradually caused by the $T$ cp of PS in MCT was higher than that of PSE in MCT.

The DSC heating curves of PS/PSE mixtures with different PS: PSE ratios in MCT are shown in Fig. $1 \mathrm{~b}$. When 5 wt $\%$ PS was added into MCT alone, the dissolution peak temperature $\left(T_{\mathrm{dp}}\right)$ of PS in MCT was 43.9 oC. When $5 \mathrm{wt} \%$ PSE added into MCT alone, the dissolution peak of PSE in MCT coincided with the melting peak of MCT due to the excellent oil solubility of PSE. With the increase of PS: PSE mass ratio, the oil solubility of PS/PSE mixtures decreased gradually, resulting in the increase of $T$ dp of PS/PSE mixtures in MCT.

The XRD patterns of PS/PSE mixtures obtained via recrystallization from MCT are illustrated in Fig. 2. It was clear that the PS: PSE mass ratios significantly affected the crystal structure of PS/PSE mixtures

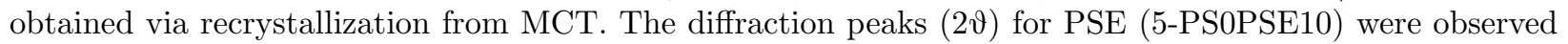
at $2 \vartheta 14.1,14.4,14.8,15.3,17.4,17.7,18.1,18.5,19.5,21.1$, and $23.6^{\mathrm{O}}$, while the respective diffraction angles for PS (5-PS10PSE0) peaks were at positions 14.2, 15.1,15.3, 16.0, 16.6,17.0,17.5, 18.0, 18.4, 18.7, $19.0,19.3,21.1,21.2$, and $21.9^{\circ}$. In contrast, when the PS: PSE mass ratio was $5: 5$, the XRD pattern of 5-PS5PSE5 was different from those of PS and PSE. The respective diffraction peak for PSE at positions $21.1^{\circ}$ and for PS at positions $16.0,17.0,18.0$ and $20.1^{\circ}$ were disappeared, indicating that a new crystal structure different from PSE and PS was generated in 5-PS5PSE5. When the PS: PSE mass ratio was lower than 5: 5, the diffraction peak patterns of PS/PSE mixtures (5-PS1PSE9 and 5-PS2PSE8) was similar with that of 5-PS0PSE10, indicating that the crystal structure of the precipitates was a mixture of new crystal structure and PSE crystal. When the PS: PSE mass ratio was higher than 5: 5, the diffraction peak patterns of PS/PSE mixtures (5-PS7PSE3 and 5-PS9PSE1) was similar with that of 5-PS10PSE0, indicating that the crystal structure of the precipitates was a mixture of new crystal structure and PS crystal.

In order to further verified that a new crystal structure different from PSE and PS was generated in PS/PSE mixtures, the melting behavior of PS/PSE mixtures obtained via recrystallization from MCT was tested by DSC. The DSC melting curves of PS/PSE mixtures with different PS: PSE mass ratios recrystallized from MCT are shown in Fig. 3. It illustrated that the melting peak temperature $(T \mathrm{mp})$ of PSE crystal recrystallized from MCT was about $70{ }^{\circ} \mathrm{C}$, while the $T \mathrm{mp}$ of PS crystal recrystallized from MCT was about $12{ }^{\circ} \mathrm{C}$. It was interesting that when the PS: PSE mass ratio was increased to 5: 5 , the $T \mathrm{mp}$ of PS/PSE mixtures obtained via recrystallization from MCT was about $113{ }^{\circ} \mathrm{C}$, which was between the $T \mathrm{mp}$ of PSE and PS crystals recrystallized from MCT, indicating that the PS/PSE mixtures precipitated from MCT formed a new crystal structure different from PSE and PS. When the PS: PSE mass ratios were lower than 5: 5, the PS/PSE mixtures recrystallized from MCT had similar melting properties with that of PSE crystals. But the $T \mathrm{mp}$ of the PS/PSE mixtures increased slightly with the increase of PS: PSE mass ratios, which indicated that the crystal structure of PS/PSE mixtures was a mixture of new crystal structure and PSE. When the PS: PSE mass ratios were higher than 5: 5, the melting curves' shape of the PS/PSE mixtures was similar with that of PS crystals, but the $T{ }_{\mathrm{mp}} \mathrm{PS} / \mathrm{PSE}$ mixtures increased with the increase of PS: PSE mass ratios, which suggested that the crystal structure of PS/PSE mixtures was a mixture of new crystal structure and PS. These results were consistent with the results of XRD, verifying that PS/PSE mixtures could form a new crystal structure different from PS and PSE when recrystallized from MCT.

The crystal morphologies of the PS/PSE mixtures obtained via recrystallization from MCT were investigated using PLM. The PLM morphologies of PS/PSE mixtures with different PS: PSE mass ratios recrystallized from MCT are presented in Fig. 4. For PSE crystals obtained via recrystallization from MCT (Fig.4a), the 
spherical crystals with average diameter of about $10 \mu \mathrm{m}$ were observed. The plate-like crystals with width of about $5 \mu \mathrm{m}$ were observed for PS crystals obtained via recrystallization from MCT (Fig.4g), which was consistent with a previous study which also showed that phytosterol exhibited plater-like morphologies in the oil media (Vaikousi, Lazaridou, Biliaderis, \& Zawistowski, 2007). It was worth noting that when the PS: PSE mass ratios were 5: 5 and 7: 3 (Fig. 4d, e), the crystal morphologies of PS/PSE mixtures obtained via recrystallization from MCT were neither spherical nor plate-like, but urchin-like crystals with a large surface area and average diameter of about $200 \mu \mathrm{m}$. When the mass ratio of PS: PSE were 1: 9 and 3: 7 (Fig. $4 \mathrm{~b}, \mathrm{c})$, the crystal morphologies of PS/PSE mixtures recrystallized from MCT were similar to that of PSE crystals, both of which exhibited spherical crystals. Furthermore, with the increase of PS: PSE mass ratios, the crystal size tended to increase. When the mass ratios of PS: PSE increased to 9:1 (Fig. 4f), the crystal morphologies of PS/PSE mixtures recrystallized from MCT were similar gradually transited to the plate-like crystals morphologies of PS, resulting in needle-like crystals morphologies with more quantity, smaller size and larger surface area. This trend was consistent with the results of Fig.2 and Fig.3.

\subsection{Recrystallization mechanism of PS/PSE mixtures in MCT}

From the above results, it could be concluded that PS/PSE mixtures had a unique recrystallization behavior in MCT, and the relevant schematic diagram is shown in Fig. 5. PS and PSE could dissolve in MCT at high temperature, and their solubility in MCT was different due to their different molecular structures. For PSE (Fig. 5a), it had lower recrystallization temperature (Fig. 1a) due to its higher solubility in MCT, and it self-assembled small number of spherical crystals structure in MCT (Fig. 4a). For PS (Fig. 5b), it has lower solubility in MCT compared with that of PSE, which was leading to the recrystallization temperature of PS in MCT was higher (Fig. 1a), and a large number of plate-like crystals were precipitated from MCT (Fig. $4 \mathrm{~g})$. It can be seen from Fig.5c, when PS and PSE coexisted in MCT at the same time, especially when the mass ratio of PS: PSE was 5: 5, PS/PSE mixtures would recrystallize into a new crystal structure in MCT (Fig. 2 and Fig. 3), showing an urchin-like crystal morphology with large size and large surface area (Fig. $4 \mathrm{~d})$.

The unique recrystallization behaviors of PS/PSE mixtures in MCT will be of great significance for controlling the quality of oils via accurately adjusting the nutritional composition of oils. Edible vegetable oils appear turbid and precipitates at low temperature (Turkulov, Dimić, Karlović, \& Vukša, 1986), which will affect consumers' acceptability of the product. Low temperature turbidity of oils is closely related to the crystallization behaviors of oils. The crystallization process of oils is divided into two processes, nucleation and growth (Himawan, Starov, \& Stapley, 2006). Crystallization nucleation is the result of metastable state after supersaturation of solution, and it is the precondition of oils' crystal growth. The common ways of oils supersaturation are cooling or adding impurities. Once the nucleus is formed, it will further develop into crystal. The introduction of impurities is one of the effective ways to promote oils crystallization, and the efficiency of impurities to promote oil crystallization depends on the structure and morphology of impurities (Fredrick et al., 2013; Kim \& Marangoni, 2017a, 2017b; Patel \& Dewettinck, 2015; Smith, Bhaggan, Talbot, \& Malssen, 2011; Talbot, Smith, \& Bhaggan, 2012). Therefore, further studies on the effects of the unique recrystallization behaviors of PS/PSE mixtures in oils on the crystallization behavior of oils is very important to control the crystallization behavior of oils by controlling the proportion of PS and PSE, so as to realize the regulation of shelf performance of oils.

\subsection{Synergistic effects of PS/PSE mixtures on promoting MCT crystallization}

PS and PSE usually coexist in natural vegetable oil, and their contents and ratios in different vegetable oils are quite different (Esche, Scholz, \& Engel, 2013; Galeano Diaz, Durán Merás, Sánchez Casas, \& Alexandre Franco, 2005). Philip et al. (Phillips, Ruggio, Toivo, Swank, \& Simpkins, 2002) showed that, generally, the total PS/PSE content in natural vegetable oils was approximately $1 \mathrm{wt} \%$, and in soybean oil, sesame oil, olive oil and cottonseed oil, PS accounted for $54-85 \%$, while in rapeseed oil, corn oil and peanut oil, PS accounted for only $32-44 \%$. It is of great significance to study the effect of PS/PSE mixtures on the crystallization behavior of oil for regulating its processing, application and storage properties. 
In order to reveal the effects of the special recrystallization behaviors of PS/PSE mixtures in oil on the crystallization behavior of oil, the influences of PS/PSE mixtures on the crystallization behavior of MCT were discussed in this study. The total content of PS and PSE was fixed to $1 \mathrm{wt} \%$, and the PS/PES/MCT mixtures with different PS: PSE mass ratios were designed. The effects of PS/PES mixtures on DSC cooling curve, $T_{\mathrm{cp}}$ and melting curve of MCT are showed in Fig. 6.

As shown in Fig. $6 \mathrm{a}$ and $6 \mathrm{~b}$, the $T$ cp of virgin MCT was $-42.2{ }^{\circ} \mathrm{C}$, and both adding 1 wt\% PSE (1PSOPSE10) or $1 \mathrm{wt} \%$ PS (1-PS10PSE0) alone could increase the $T \mathrm{cp}$ of MCT to $-40.5{ }^{\circ} \mathrm{C}$ and $-32.7{ }^{\circ} \mathrm{C}$, respectively. Compared PSE, the hydroxyl group of PS molecule leads to the poor compatibility between PS and triglyceride (Vaikousi, Lazaridou, Biliaderis, \& Zawistowski, 2007), and thus PS is more easily to recrystallize from MCT (Fig.1a), acting as nucleating agents to increase the $T_{\mathrm{cp}}$ of MCT, which resulted in PS was more efficient than PSE in promoting MCT crystallization. It was also demonstrated from Fig. $6 \mathrm{a}$ and $6 \mathrm{~b}$ that $1 \mathrm{wt} \%$ PS/PSE mixtures could significantly increase the $T$ cp of MCT, and $1 \mathrm{wt} \% \mathrm{PS} / \mathrm{PSE}$ mixtures with all PS: PES mass ratios had higher efficiency in promoting MCT crystallization than 1 wt\% PSE. With the increase of PS: PSE mass ratios, the $T_{\mathrm{cp}}$ of MCT increased gradually. When the mass ratio of PS: PSE increased to 5: 5 or above, the $T$ cp of MCT was higher than that of MCT with 1 wt\% PSE and MCT with $1 \mathrm{wt} \%$ PS, which indicated that PS and PSE had synergistic effect on the crystallization behavior of MCT. For example, when the mass ratio of PS: PES was 5: 5, the $T$ cp of 1-PS5PSE5 was $-30.2{ }^{\circ} \mathrm{C}, 10.3$ ${ }^{\mathrm{o}} \mathrm{C}$ higher than that of 1-PS0PSE10 and $2.5{ }^{\circ} \mathrm{C}$ higher than that of 1-PS10PSE0.

Fig. 6c illustrated that the PSE, PS and PS/PSE mixtures had a great influence on the DSC melting curve of MCT. For virgin MCT, there was a small exothermic peak at $-32.5^{\circ} \mathrm{C}$ appeared. It might be attributed to that MCT could not be completely crystallized when the temperature drops to $-75{ }^{\circ} \mathrm{C}$; with the temperature rising to - 32.5, the non-crystallized MCT continued to crystallize leading to the exothermic peak appeared. When PSE, PS or PS/PSE mixtures was added, the crystallization of MCT was completed in the cooling process due to the crystallization-promoting effect of PSE, PS or PS/PSE mixtures, thus the exothermic peak at - 32.5 oC disappeared.

PLM was used to further verify that PS and PSE could synergistic promote the crystallization of MCT. The PLM non-isothermal crystallization morphologies of virgin MCT, 1-PS0PSE10, 1-PS10PSE0 and 1PS5PSE5 are displayed in Fig. 7. For virgin MCT (Fig. 7a), its initial crystallization temperature was approximately - 30 oC. For 1-PS0PSE10, its initial crystallization temperature was - 28 oC, higher than that of virgin MCT, and the number of crystals was also more than that of virgin MCT. For 1-PS10PSE0, its initial crystallization temperature was - $27 \mathrm{oC}$, higher than that of virgin MCT and 1-PS0PSE10, and the number of crystals was increased obviously compared with that of virgin MCT and 1-PSOPSE10. These results suggested that both PSE and PS could promote the crystallization nucleation of MCT, but PSE was less effective than PS. While for 1-PS5PSE5, it had a lowest initial crystallization temperature of - 20 oC among four samples, which indicated that the PS/PSE mixtures showed the strongest nucleation effect. In addition, the crystal size of 1-PS5PSE5 at - 25 oC was bigger than 1-PS0PSE10 at - 28 oC and 1-PS10PSE0 at - 27 oC. PS and PSE could be dissolved in MCT at high temperature and recrystallized in MCT at low temperature, which could be using as nucleating agents to promote the crystallization of MCT. However, the nucleation efficiencies of the recrystallized PS and PSE crystals were directly related to their crystal morphologies. Since PSE had higher solubility in MCT, it would precipitate from MCT at relative low temperature in the form of a small amount of spherical crystals (Fig. 4a), and the initial crystallization temperature of MCT increased slightly. Compared with PSE, PS had lower solubility in MCT and it would recrystallize from MCT at relative high temperature in the form of a large number of plate-like crystals (Fig. $4 \mathrm{~g}$ ), which had higher nucleation efficiency, thus the initial crystallization temperature of MCT increased greatly. When PS and PSE were used together, the PS/PSE mixtures could recrystallize a new crystal structure in form of special urchin-like aggregation morphology with larger nucleation surface (Fig. 4d), which made the initial crystallization temperature of MCT increased the most compared to that of using PS or PSE alone. These results were consistent with the above results, which further indicated that the special recrystallization behavior of PS/PSE mixtures in MCT synergistically promoted the crystallization of MCT. These results will be helpful to control the crystallization behavior of oil by precisely controlling the content 
and mass ratio of PS/PSE mixtures.

\section{Conclusion}

The crystallization temperature, crystal form and crystal morphology of PS/PSE mixtures in MCT were studied by DSC, XRD and PLM. The results showed that the crystallization peak temperature of PS/PSE mixtures in MCT decreased first and then increased with the increase of PS: PSE mass ratio, while the dissolution peak temperature of PS/PSE mixtures in MCT increased gradually with the increase of PS: PSE mass ratio. When the mass ratio of PS: PSE was greater than 3: 7, PS/PSE mixtures precipitated a new urchin-like crystal structure, different from PS spherical or PSE plate-like crystal structure, in MCT. The new urchin-like PS/PSE crystals had a larger surface area, and its melting point was between PS and PSE. Furthermore, the effects of PS/PSE mixtures on the crystallization behavior of MCT were investigated. The results indicated that PS/PSE mixtures could synergistically promote the crystallization of MCT when the PS: PSE mass ratio was greater than 3:7. When the total content of PS and PSE was $1 \mathrm{wt} \%$, and the mass ratio of PS: PSE was 5: 5, the crystallization peak temperature of PS/PSE/MCT was 12 oC higher than that of virgin MCT, $2.5 \mathrm{oC}$ higher than that of PS/MCT, and $10.3 \mathrm{oC}$ higher than that of PSE/MCT. These results not only provide a method for obtaining new crystal structure of phytosterols, but also have great significance for controlling the quality of oils by precisely adjusting the nutritional composition of oils.

\section{Acknowledgements}

The authors thank the Shanghai Rising-Star Program (Grant NO.21QB1400200) and National Natural Science Funds of China (Grant NO.21606084) for financial support.

\section{Declaration of Competing Interest}

The authors declare that they have no known competing financial interests or personal relationships that could have appeared to influence the work reported in this paper.

\section{References}

Bin Sintang, M. D., Rimaux, T., Van de Walle, D., Dewettinck, K., \& Patel, A. R. (2017). Oil structuring properties of monoglycerides and phytosterols mixtures. European Journal of Lipid Science and Technology , 119 , 1-14. https://doi.org/10.1002/ejlt.201500517.

Bohn, T., Tian, Q., Chitchumroonchokchai, C., Failla, M. L., Schwartz, S. J., Cotter, R., \& Waksman, J. A. (2007). Supplementation of test meals with fat-free phytosterol products can reduce cholesterol micellarization during simulated digestion and cholesterol accumulation by Caco-2 cells. Journal of agricultural and food chemistry ,55 , 267-272. https://doi.org/10.1021/jf0618291.

Bouic, P., Clark, A., Lamprecht, J., Freestone, M., Pool, E., Liebenberg, R., Kotze, D., \& Jaarsveld, P. (1999). The effects of B-sitosterol (BSS) and B-sitosterol glucoside (BSSG) mixture on selected immune parameters of marathon runners: inhibition of post marathon immune suppression and inflammation. International Journal of Sports Medicine, 20 , 258-262. https://doi.org/10.1055/s-2007-971127.

Daels, E., Foubert, I., \& Goderis, B. (2017). The effect of adding a commercial phytosterol ester mixture on the phase behavior of palm oil.Food Research International , 100 , 841-849. https://doi.org/10.1016/j.foodres.2017.08.015.

Esche, R., Scholz, B., \& Engel, K.-H. (2013). Online LC-GC analysis of free sterols/stanols and intact steryl/stanyl esters in cereals.Journal of Agricultural and Food Chemistry , 61 , 10932-10939. https://doi.org/10.1021/jf403046z.

Fredrick, E., Moens, K., Heyman, B., Fischer, S., Meeren, P. V. D., \& Dewettinck, K. (2013). Monoacylglycerols in dairy recombined cream: I. The effect on milk fat crystallization. Food Research International, 51 , 892-898. https://doi.org/10.1016/j.foodres.2013.02.007. 
Galeano Diaz, T., Duran Meras, I., Sanchez Casas, J., \& Alexandre Franco, M. F. (2005). Characterization of virgin olive oils according to its triglycerides and sterols composition by chemometric methods. Food Control, 16 , 339-347. https://doi.org/10.1016/j.foodcont.2004.03.014.

Goldberg, A. C., Ostlund, R. E., Bateman, J. H., Schimmoeller, L., McPherson, T. B., \& Spilburg, C. A. (2006). Effect of plant stanol tablets on low-density lipoprotein cholesterol lowering in patients on statin drugs. The American Journal of Cardiology , 97 , 376-379. https://doi.org/10.1016/j.amjcard.2005.08.056.

Hannan, M. A., Sohag, A. A. M., Dash, R., Haque, M. N., Mohibbullah, M., Oktaviani, D. F., Hossain, M. T., Choi, H. J., \& Moon, I. S. (2020). Phytosterols of marine algae: Insights into the potential health benefits and molecular pharmacology. Phytomedicine ,69 , 1-19. https://doi.org/10.1016/j.phymed.2020.153201.

Himawan, C., Starov, V. M., \& Stapley, A. G. F. (2006). Thermodynamic and kinetic aspects of fat crystallization. Advances in Colloid and Interface Science, 122 , 3-33. https://doi.org/10.1016/j.cis.2006.06.016.

Kim, G. Y., \& Marangoni, A. (2017a). Crystallization behavior of high behenic acid stabilizers in liquid oil. Journal of the American Oil Chemists' Society , 94, 1165-1173. https://doi.org/10.1007/s11746-017-3021-z.

Kim, G. Y., \& Marangoni, A. (2017b). Engineering the nucleation of edible fats using a high behenic acid stabilizer. European Journal of Lipid Science and Technology , 119 , 1-13. https://doi.org/10.1002/ejlt.201700154.

Nguyen, H. C., Huang, K.-C., \& Su, C.-H. (2020). Green process for the preparation of phytosterol esters: Microwave-mediated noncatalytic synthesis. Chemical Engineering Journal , 382 , 1-8. https://doi.org/10.1016/j.cej.2019.122796.

Patel, A. R., \& Dewettinck, K. (2015). Current update on the influence of minor lipid components, shear and presence of interfaces on fat crystallization. Current Opinion in Food Science, 3,65-70. https://doi.org/10.1016/j.cofs.2015.05.010.

Phillips, K. M., Ruggio, D. M., Toivo, J. I., Swank, M. A., \& Simpkins, A. H. (2002). Free and esterified sterol composition of edible oils and fats. Journal of Food Composition and Analysis, 15 , 123-142. https://doi.org/10.1006/jfca.2001.1044.

Sawalha, H., den Adel, R., Venema, P., Bot, A., Floter, E., \& van der Linden, E. (2012). Organogel-emulsions with mixtures of $\beta$-sitosterol and $\gamma$-oryzanol: Influence of water activity and type oil phase on gelling capability. Journal of Agricultural and Food Chemistry ,60 , 3462-3470. https://doi.org/10.1021/jf300313f.

Sawalha, H., Venema, P., Bot, A., Flöter, E., \& van der Linden, E. (2011). The influence of concentration and temperature on the formation of $\gamma$-oryzanol $+\beta$-sitosterol tubules in edible oil organogels.Food Biophysics, 6 , 20-25. https://doi.org/10.1007/s11483-010-9169-9.

Shahzad, N., Khan, W., Md, S., Ali, A., Saluja, S. S., Sharma, S., Al-Allaf, F. A., Abduljaleel, Z., Ibrahim, I. A. A., Abdel-Wahab, A. F., Afify, M. A., \& Al-Ghamdi, S. S. (2017). Phytosterols as a natural anticancer agent: Current status and future perspective.Biomedicine $\&$ Pharmacotherapy , 88 , 786-794. https://doi.org/10.1016/j.biopha.2017.01.068.

Smith, K., Bhaggan, K., Talbot, G., \& Malssen, K. (2011). Crystallization of fats: Influence of minor components and additives.Journal of the American Oil Chemists' Society , 88,1085-1101. https://doi.org/10.1007/s11746-011-1819-7.

Talbot, G., Smith, K., \& Bhaggan, K. (2012). Influence of minor components on fat crystallization. Lipid Technology ,24,83-85. https://doi.org/10.1002/lite.201200180.

Turkulov, J., Dimić, E., Karlović, D., \& Vukša, V. (1986). The effect of temperature and wax content on the appearance of turbidity in sunflowerseed oil. Journal of the American Oil Chemists' Society ,63 , 1360-1363. https://doi.org/10.1007/BF02679602. 
Vaikousi, H., Lazaridou, A., Biliaderis, C., \& Zawistowski, J. (2007). Phase transitions, solubility, and crystallization kinetics of phytosterols and phytosterol-oil blends. Journal of Agricultural and Food Chemistry , 55 , 1790-1798. https://doi.org/10.1021/jf0624289.

Vu, P.-L., Shin, J.-A., Lim, C.-H., \& Lee, K.-T. (2004). Lipase-catalyzed production of phytosteryl ester and their crystallization behavior in corn oil. Food Research International , 37, 175-180. https://doi.org/10.1016/j.foodres.2003.11.005.

Wester, I. (2000). Cholesterol-lowering effect of plant sterols.European Journal of Lipid Science and Technology , 102 , 37-44. https://doi.org/10.1002/(SICI)1438-9312(200001)102:1\%3C37::AIDEJLT37\%3E3.0.CO;2-1.

Yang, F., Oyeyinka, S. A., \& Ma, Y. (2016). Novel synthesis of phytosterol ester from soybean sterol and acetic anhydride.Journal of Food Science, 81, 1629-1635. https://doi.org/10.1111/1750-3841.13354.

\section{Figure captions}

Fig. 1 DSC cooling (a) and heating (b) profiles of PS/PSE mixtures in MCT

Fig. 2 XRD patterns of PS/PSE mixtures obtained via recrystallization from MCT

Fig. 3 DSC melting curves of PS/PSE mixtures obtained via recrystallization from MCT

Fig. 4 PLM morphologies of PS/PSE mixtures obtained via recrystallization from MCT

Fig. 5 Recrystallization processing of PS/PSE mixtures in MCT

Fig. 6 DSC cooling curves (a), $T_{\text {cp }}$ (b) and melting curves (c) of MCT with PS/PES mixtures

Fig. 7 PLM morphologies of pure MCT (a), 1-PS10PSE0 (b), 1-PS0PSE10 (c) and 1-PS5PSE5 (d) 

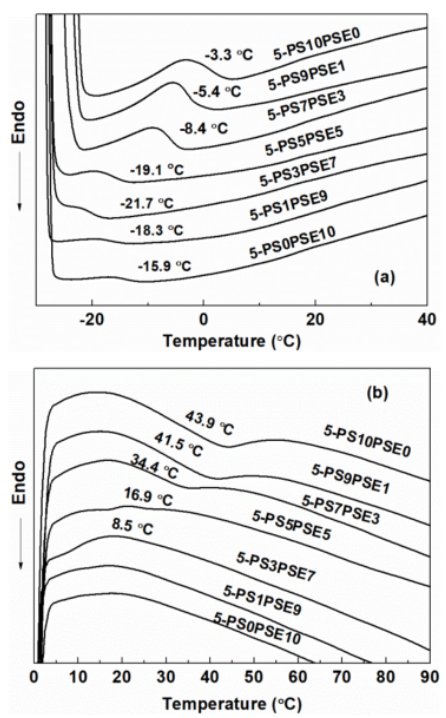

Fig. 1 DSC cooling (a) and heating (b) profiles of PS/PSE mixtures in MCT 


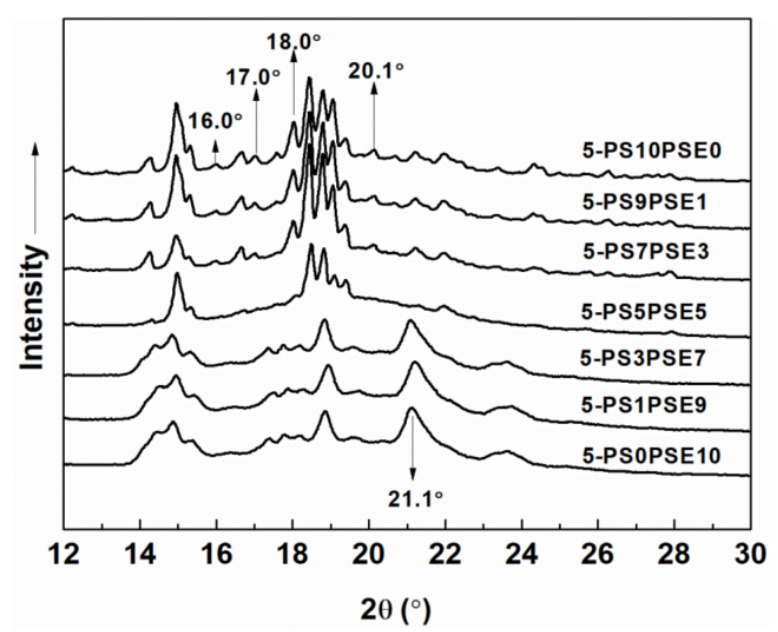

Fig. 2 XRD patterns of PS/PSE mixtures obtained via recrystallization from MCT 


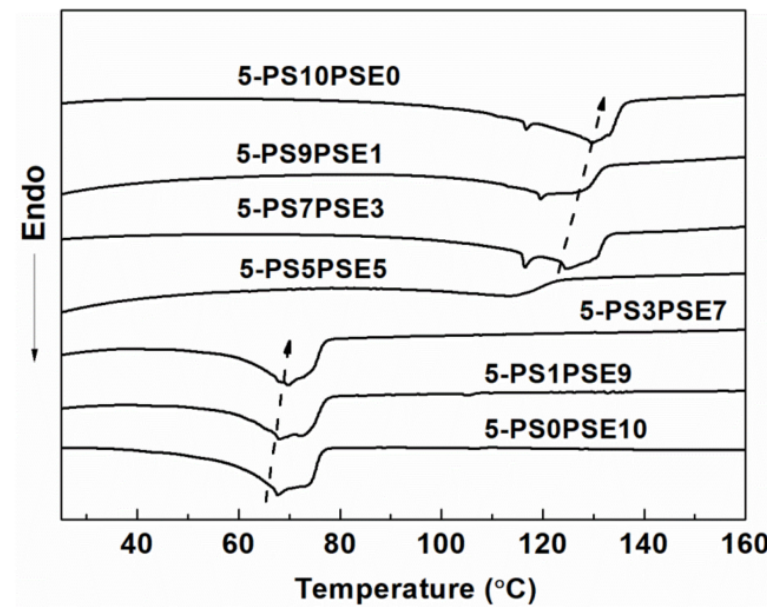

Fig. 3 DSC melting curves of PS/PSE mixtures obtained via recrystallization from MCT 


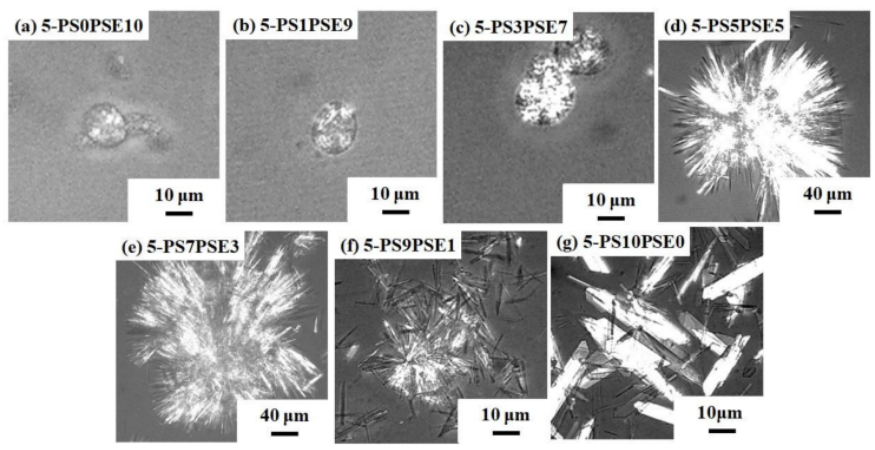

Fig. 4 PLM morphologies of PS/PSE mixtures obtained via recrystallization from MCT 
(a) PSE/MCT

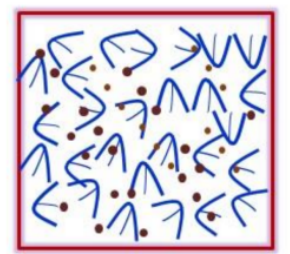

$\sqrt{3}$

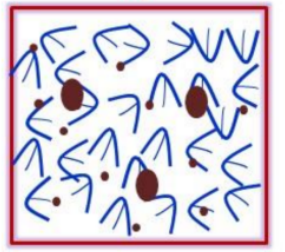

$\leqslant$ TAG molecule

PS crystal (b) PS/MCT

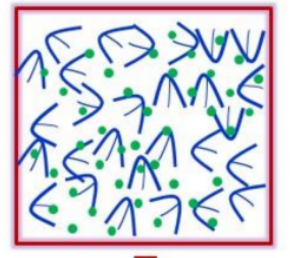

ก

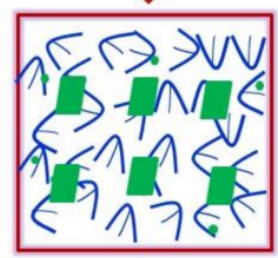

- PS molecule

PSE crystal (c) PS/PSE/MCT

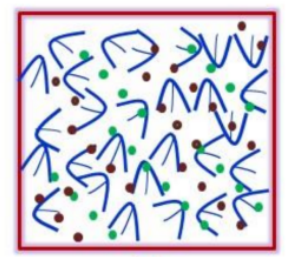

ก

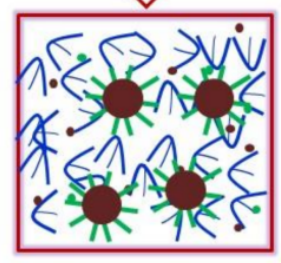

- PSE molecule

PS/PSE crystal

Fig. 5 Recrystallization processing of PS/PSE mixtures in MCT 

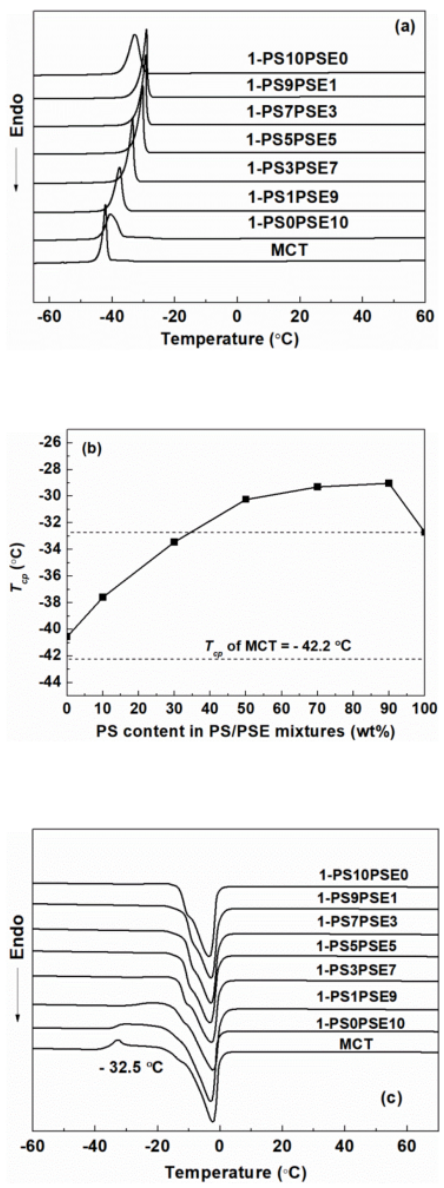

Fig. 6 DSC cooling curves (a), $T_{\mathrm{cp}}$ (b) and melting curves (c) of MCT with PS/PES mixtures 


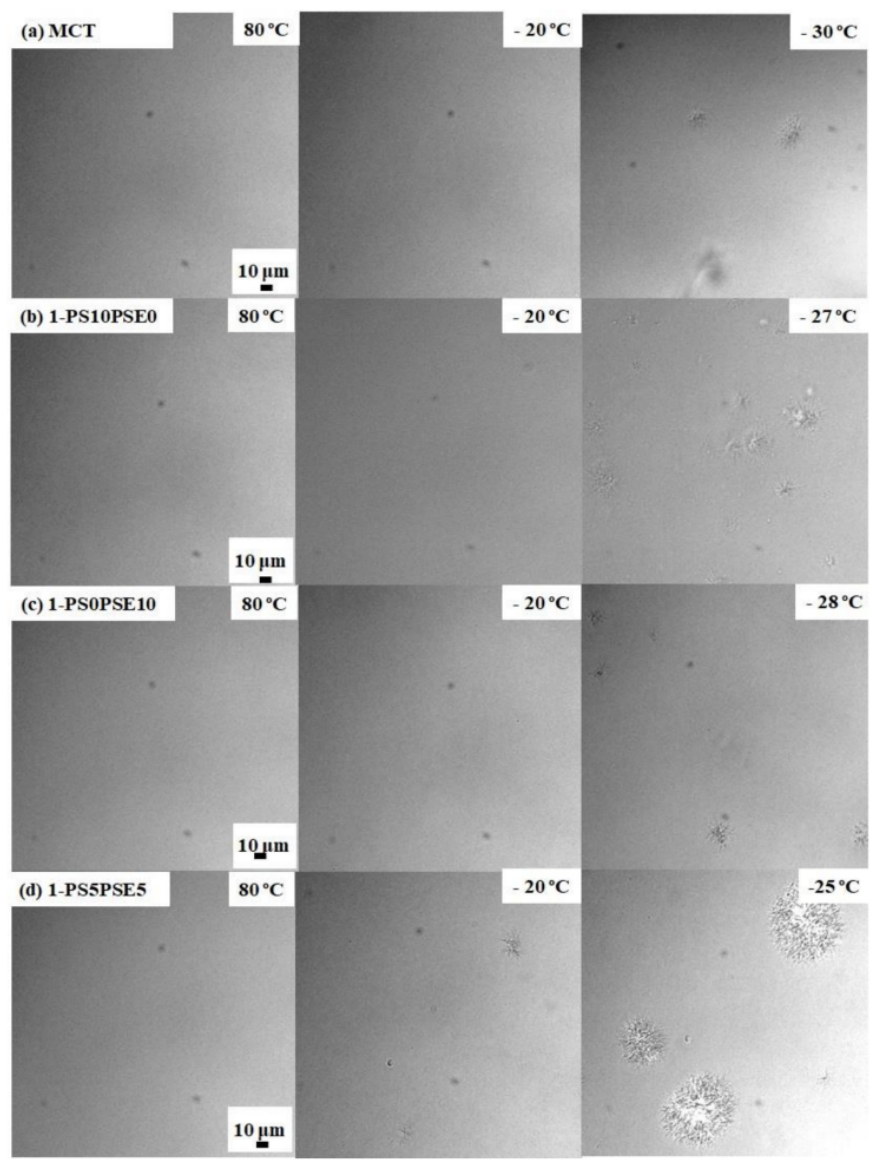

Fig. 7 PLM morphologies of pure MCT (a), 1-PS10PSE0 (b), 1-PS0PSE10 (c) and 1-PS5PSE5 (d) 